\title{
THE
}

$9-1-1971$

\section{\-Nucleon Charge-Symmetry-Breaking Interaction. II. Rank-2 Potentials}

\author{
E. Sullivan
}

Kenneth L. Hartt

University of Rhode Island, hartt@uri.edu

Follow this and additional works at: https://digitalcommons.uri.edu/phys_facpubs

Terms of Use

All rights reserved under copyright.

\section{Citation/Publisher Attribution}

Sullivan, E., \& Hartt, K. (1971). ^-Nucleon Charge-Symmetry-Breaking Interaction. II. Rank-2 Potentials. Physical Review D, 4(5), 1366. doi: 10.1103/PhysRevD.4.1366

Available at: http://dx.doi.org/10.1103/PhysRevD.4.1366

This Article is brought to you for free and open access by the Physics at DigitalCommons@URI. It has been accepted for inclusion in Physics Faculty Publications by an authorized administrator of DigitalCommons@URI. For more information, please contact digitalcommons-group@uri.edu. 
Physics and Nuclear Structure, edited by G. Alexander (North-Holland, Amsterdam, 1967), pp. 60-89.

${ }^{31}$ We employ a definition of scattering length, $a$, and effective range, $\mathrm{r}_{0}$, which is consistent with $k \cot \delta \approx$ $-a^{-1}+\frac{1}{2} r_{0} k^{2}$.

${ }^{32}$ R. C. Herndon, Y. C. Tang, and G. W. Schmid, Phys. Rev. 137, B294 (1965).

${ }^{33}$ Y. C. Tang, Ref. 9, p. 276.
${ }^{34}$ B. Povh II, invited talk at the New York meeting of the American Physical Society, 1971 (unpublished).

${ }^{35}$ P. M. Morse and H. Feshbach, Methods of Theoretical Physics, Part II (McGraw-Hill, New York, 1953), p. 1137.

${ }^{36}$ We are grateful to Dr. J. S. Levinger and Dr. R. Stagat for furnishing us with information about the application of this method.

\title{
$\Lambda$-Nucleon Charge-Symmetry-Breaking Interaction. II. Rank-2 Potentials
}

\author{
E. Sullivan* and K. Hartt \\ Department of Physics, University of Rhode Island, Kingston, Rhode Island 02881 \\ (Received 8 September 1969; revised manuscript received 17 May 1971)
}

\begin{abstract}
A potential model of the $\Lambda-N$ interaction containing short-range repulsions is constructed to study charge-symmetry-breaking (CSB). Rank-2 nonlocal central potentials and a rank-1 approximation to them (referred to as UPAA) are required to fit low-energy $\Lambda-p$ scattering cross sections, the $\Lambda$-separation energy $\left(B_{\Lambda}\right)$ from ${ }_{\Lambda} \mathrm{H}^{3}$, and a dimensionless parameter which partially characterizes the CSB part of the binding-energy difference between ${ }_{\Lambda} \mathrm{H}^{4}$ and ${ }_{\Lambda} \mathrm{He}^{4}$. In a scan of these data, a class of potentials with acceptable $\chi^{2}$ fits is found. Repulsions are seen to reduce significantly the lower limits of the singlet and triplet CSB strengths required in the entire data scan, from the CSB found earlier without including repulsions. In particular, for the largest value of $B_{\Lambda}$ used, $0.25 \mathrm{MeV}$, qualitative agreement with Downs's $\mathrm{SU}_{3}$ model of CSB is attained, in that the $\Lambda-p$ CSB singlet interaction can be repulsive. This conclusion also holds for the UPAA, with an indication that a short-range weakening of $\Lambda-N$ attraction has an effect similar to a $\Lambda-N$ repulsion. Effects of isospin mixing in ${ }_{\Lambda} \mathrm{H}^{3}$ are taken into account. The $\Lambda-N$ potentials resulting from this analysis are seen to vary in a systematic way within the statistical spread of available data.
\end{abstract}

\section{INTRODUCTION}

In the preceding paper ${ }^{1}$ (henceforth referred to as I), we have sought limitations upon the chargesymmetry-breaking (CSB) component of the $\Lambda-N$ interaction as imposed by experiment. Adopting the notation of $\mathrm{I}$, the $\Lambda-N$ two-body CSB interaction is written

$$
W_{C S B}=-\tau_{3}(N)\left(\lambda_{B} / 2 \mu_{\Lambda N}\right)[\alpha+\vec{\sigma}(\Lambda) \cdot \vec{\sigma}(N)] W_{p},
$$

where $\tau_{3}(N)$ is a Pauli isospin operator, $\lambda_{B}$ is an over-all interaction strength, $\alpha$ is a spin-independent constant, and $\mu_{\Lambda N}$ is the $\Lambda-N$ reduced mass. $W_{p}$ contains the momentum dependence of the potential, and is defined to be positive in the limit of low relative momenta. The singlet and triplet CSB strength $s$ in the $\Lambda-p$ state, denoted by $\epsilon_{s}$ and $\epsilon_{t}$, are $\epsilon_{s}=\lambda_{B}(\alpha-3)$ and $\epsilon_{t}=\lambda_{B}(\alpha+1)$. Using two-, three-, and four-baryon data, and a rank-1 centralseparable-potential model in which all potentials, including $W_{p}$, have Yamaguchi shape, ${ }^{2}$ we found in I a class of potentials which gave good $\chi^{2}$ fits to all data. One of our results was to find only attractive $\Lambda-p$ CSB contributions present, expressed by the inequalities $\epsilon_{s}>0, \epsilon_{t}>0$. This disagrees with one of the predictions of the $\mathrm{SU}_{3}$ particle-mixing model of Downs, ${ }^{3}$ that the long-range part of the $\Lambda-p$ CSB singlet interaction should produce a repulsion.

The purpose of the present paper is to test the conclusions of I by extending the analysis to a class of potentials with different shapes. In order to study the effect of short-range repulsions in the $\Lambda-N$ system, we introduce rank-2 interactions. As seen in Secs. II and III, we are thereby able to produce a change in sign of the $S$-wave phase shifts both in the $\Lambda-N$ and the $N-N$ interactions, which is indicative of strong short-range repulsions, and come to better agreement with some of the well-known experimental results for the $\mathrm{N}-\mathrm{N}$ interaction.

Potentials that have a two-body state which is just bound or just unbound are frequently well approximated in the low-energy regime by rank-1 potentials, in what has come to be known as the unitary pole approximation (UPA). ${ }^{4,5}$ We introduce here a closely related approximation, which we refer to as the UPAA. We have in mind the dual purpose of testing the pole approximation to the rank-2 potentials introduced here and studying 
the effects of still other types of short-range behavior in few-baryon systems.

In this phenomenological analysis we carry further our study, begun in I, of isospin mixing in ${ }_{\Lambda} \mathrm{H}^{3}$ and of the possible consequences of a spin assignment, $I=1$, for the hypernucleus ${ }_{\Lambda} \mathrm{He}^{4}$. The more commonly assumed value, $I=0$, is also included. As in I, we continue with a one-channel formalism in which the $\Sigma$ state does not occur explicitly in the wave function, and we make the following assumptions:

(a) The two-body $S$-wave $\Lambda-N$ and $N-N$ interactions employed adequately describe the states and the energies studied.

(b) The intrinsic range $b$ is taken to be the same for the CS and CSB parts of the $\Lambda-N$ interaction.

(c) Tensor $\Lambda-N$ and $N-N$ terms present in the full interaction are represented by effective central triplet terms.

(d) The experimental CSB effect seen in the binding-energy difference ${ }^{6}$ between ${ }_{\Lambda} \mathrm{He}^{4}$ and ${ }_{\Lambda} \mathrm{H}^{4}$ is partially represented by a dimensionless parameter, $\Delta$, defined by

$$
\Delta=\frac{\lambda_{n p p}-\lambda_{n n p}}{\lambda_{n p p}+\lambda_{n n p}}
$$

where $\lambda_{n p p}$ and $\lambda_{n n p}$ are, respectively, the spinaveraged $\Lambda-N$ interaction strengths in ${ }_{\Lambda} \mathrm{He}^{4}$ and ${ }_{\Lambda} \mathrm{H}^{4}$. We take the value $\Delta=0.01$ which we extract from the results of Herndon and Tang ${ }^{7}$ as a portion of the experimental data to be fitted. As pointed out in $\mathrm{I}$, this value of $\Delta$ should be more realistic for a model in which short-range repulsions are present.

In addition to $\Delta$, the data include six points of the total $\Lambda-p$ cross section, $\sigma(\Lambda p)$, with $E_{\text {c.m. }}<18$ $\mathrm{MeV}$. This was observed in I to be almost entirely $S$ wave. Also the $\Lambda$ binding energy of ${ }_{\Lambda} \mathrm{H}^{3}$ is allowed a spread of values, $B_{\Lambda}=0.05,0.17$, and $0.25 \mathrm{MeV}$, reflecting the current uncertainty about this number. ${ }^{6-8}$

Our rank-2 potentials are taken as differences between Yamaguchi potentials. In addition to creating an analytically simple representation, this choice is shown in Sec. III to lead to an excellent representation of $n-p$ data. The rank-2 and also the UPAA interactions are used to compute $B_{\Lambda}$ to high numerical precision, and the results are included in an on-line self-consistent calculation which is based on a scan of acceptable fits to $\sigma(\Lambda p)$ subject to the four-baryon constraint met by requiring $\Delta=0.01$. The results of this self-consistent procedure are discussed in Secs. III and IV.

\section{RANK-2 POTENTIALS, THE UPA, AND THE UPAA}

This section begins with a brief review of some formal results needed for the definition of our po- tentials, including the UPA, which is employed as an alternative description of the $n-p$ and $\Lambda-N$ interactions. It introduces a modified UPA, referred to as the UPAA, which has been found satisfactory for the $\Lambda-N$ interactions studied in this paper. Details of constructing an $n$ - $p$ potential which is suitable for the model of the hypertriton employed in this paper conclude this section. Natural units, with $\hbar=c=1$, are used throughout the paper.

A general expression for a central rank-2 potential is

$\left\langle\overrightarrow{\mathrm{k}}|V| \overrightarrow{\mathrm{k}}^{\prime}\right\rangle=-\left(\lambda_{A} / 2 \mu\right) g_{A}(k) g_{A}\left(k^{\prime}\right)+\left(\lambda_{R} / 2 \mu\right) g_{R}(k) g_{R}\left(k^{\prime}\right)$,

where $\lambda_{A}$ and $\lambda_{R}$ are the attractive and repulsive interaction strengths [provided both $\lambda_{A}$ and $\lambda_{R}$ as well as $g_{A}(k)$ and $g_{R}(k)$ are positive $], g_{A}(k)$ and $g_{R}(k)$ are the corresponding form factors, and $\mu$ is the reduced mass of the two particles. From Tabakin, ${ }^{9}$ the associated $t$ matrix takes the form

$$
\left\langle\overrightarrow{\mathrm{k}}|t(E)| \overrightarrow{\mathrm{k}}^{\prime}\right\rangle=N / D,
$$

where

$$
\begin{aligned}
& N=-g_{A}\left(k^{\prime}\right) g_{A}(k)\left(\lambda_{R}^{-1}-M\right)+g_{R}\left(k^{\prime}\right) g_{R}(k)\left(\lambda_{A}^{-1}+J\right) \\
& -\left[g_{A}\left(k^{\prime}\right) g_{R}(k)+g_{A}(k) g_{R}\left(k^{\prime}\right)\right] L, \\
& D=2 \mu\left[\left(\lambda_{R}^{-1}-M\right)\left(\lambda_{A}^{-1}+J\right)+L^{2}\right],
\end{aligned}
$$

and

$$
\begin{aligned}
& J(E)=\int d^{3} k g_{A}{ }^{2}(k)\left(2 \mu E-k^{2}\right)^{-1}, \\
& L(E)=\int d^{3} k g_{A}(k) g_{R}(k)\left(2 \mu E-k^{2}\right)^{-1}, \\
& M(E)=\int d^{3} k g_{R}{ }^{2}(k)\left(2 \mu E-k^{2}\right)^{-1}
\end{aligned}
$$

In addition to yielding directly the scattering amplitude, the $t$ matrix of Eq. (4) contains information about bound states, or nearly bound states, through its singularities. ${ }^{5,9}$ If $B$ is the two-body binding energy, then

$$
\frac{D(-B)}{2 \mu}=0=\left[\lambda_{A}^{-1}+J(-B)\right]\left[\lambda_{R}^{-1}-M(-B)\right]+L^{2}(-B),
$$

where $\sqrt{-B}$ corresponds to the physical state on the $k$ plane. If there is no bound state, $-\sqrt{-B}$ locates a singularity on the $k$ plane corresponding to a nonphysical or virtual state.

The UPA provides a prescription for finding a rank-1 approximation to a potential such as given in Eq. (3). One requires that the UPA yield the same wave function as the potential being approximated at an energy where the $t$ matrix is singular. The UPA is then expected to provide a good approx- 
imation to the actual $t$ matrix for energies in the neighborhood of this singularity. ${ }^{4,5,9}$ Following Brady et al., the UPA corresponding to Eq. (3) takes the form

$$
\left\langle\vec{k}|V| \vec{k}^{\prime}\right\rangle_{\mathrm{UPA}}=\left(\lambda_{u} / 2 \mu\right) g_{u}(k) g_{u}\left(k^{\prime}\right),
$$

where

$$
g_{u}(k)=g_{A}(k)+\frac{L(-B)}{\lambda_{R}{ }^{-1}-M(-B)} g_{R}(k)
$$

and

$$
\lambda_{w^{-1}}=\lambda_{A}{ }^{-1}-\lambda_{R}{ }^{-1} \frac{L^{2}(-B)}{\left[\lambda_{R}^{-1}-M(-B)\right]^{2}} .
$$

The UPA $t$ matrix becomes

$$
\left\langle\overrightarrow{\mathrm{k}}\left|t_{u}(E)\right| \overrightarrow{\mathrm{k}}^{\prime}\right\rangle=\frac{-g_{u}(k) g_{u}\left(k^{\prime}\right)}{2 \mu\left[\lambda_{u}{ }^{-1}+J_{u}(E)\right]},
$$

with

$$
\begin{aligned}
J_{u}(E)= & J(E)+\frac{2 L(-B)}{\lambda_{R}^{-1}-M(-B)} L(E) \\
& +\frac{L^{2}(-B)}{\left[\lambda_{R}^{-1}-M(-B)\right]^{2}} M(E) .
\end{aligned}
$$

The shape of the UPA form factor given in Eqs. (9)-(11) is dependent upon the value of $B$ as well as the analytic properties of the form factors $g_{A}(k)$ and $g_{R}(k)$. Hence, if one starts out with a rank-2 model of singlet and triplet $\Lambda-N$ interactions which employ common form factors, $g_{A}(k)$ and $g_{R}(k)$, their UPA form factors will generally not have the same shape.

From the computational standpoint, it is a significant simplification to maintain equal shapes for the $\Lambda-N$ singlet and triplet interactions when studying ${ }_{\Lambda} \mathrm{H}^{3}$. It is therefore gratifying to see that such a condition can be fulfilled to a good approximation here. The UPAA is a prescription which forces the form factors to be the same if the rank2 form factors are equal, and is therefore an approximation to the UPA. Such an approximation is employed in conjunction with the rank-2 $\Lambda-N$ interactions of this paper. The best data fits in the $\Lambda-N$ case are obtained by forcing the triplet interaction, which is the weaker, to assume the shape of the UPA for the singlet.

The pole condition for the singlet UPA $\Lambda-N$ interaction becomes

$$
\lambda_{u s}{ }^{-1}+J_{u s}(-B)=0,
$$

where the subscript label $s$ is used to identify singlet-interaction quantities. Also, from Eq. (13),

$$
J_{u s}(E)=J_{s}(E)-2 X_{s} L_{s}(E)+X_{s}^{2} M_{s}(E),
$$
with

$$
X_{s}=\frac{L_{s}(-B)}{M_{s}(-B)-\lambda_{R s}{ }^{-1}} .
$$

For the UPA, equations identical to Eqs. (14)-(16) hold for the triplet quantities, $\lambda_{u t}, J_{u t}, J_{t}, L_{t}$, $M_{t}, \lambda_{R t}$, and $X_{t}$. However, the UPAA requires setting $X_{t}$ equal to $X_{s}$. A new value of $\lambda_{u t}$ is then found from Eq. (11), viz.,

$$
\lambda_{u t}=\left(\lambda_{A t}{ }^{-1}-\lambda_{R t}{ }^{-1} X_{s}^{2}\right)^{-1} \text {. }
$$

The applications of these formulas involve two distinct cases. The $n-p$ rank- 2 potential contains different form factors in the singlet and triplet states. Here, the straight UPA is used. Only for the $\Lambda-N$ interaction, where the singlet and triplet form factors in the original rank-2 expressions are assumed to be the same, is the UPAA employed. In the $\Lambda-N$ case, then, the rank-1 singlet and triplet form factors are identical.

For the $\Lambda-N$ case, using $X_{s}$ rather than the UPA value, $X_{t}$, in Eq. (17) gives a value for $\lambda_{u t}$ which tends to compensate for the error in the location of the singularity caused by the adjustment of $X_{t}$. This is easily shown in the case where $J_{u}$ is a monotonic function of $E$, which is true for the rank2 potentials employed here. Table I shows a comparison, for some typical $\Lambda-N$ potentials, between the rank-2 effective-range parameters and the two associated approximations, the UPA and the UPAA. For the interactions studied in this paper, the UPAA is in fact slightly better than the UPA very low energies.

The $\Lambda-N$ form factors are taken to be of the Yamaguchi ${ }^{2}$ shape:

$$
g_{A}(k)=\left(k^{2}+\beta^{2}\right)^{-1}, \quad g_{R}(k)=\left(k^{2}+\gamma^{2}\right)^{-1} .
$$

This is also the shape of form factor chosen for the $n-p$ potential. To distinguish them from the $\Lambda-N$ case, the $n-p$ form factors are expressed as $f_{A}(k)$ and $f_{R}(k)$.

The $n$ - $p$ attractive and repulsive range parameters, $\beta$ and $\gamma$, and the $n-p$ strengths $\lambda_{A}$ and $\lambda_{R}$, are shown in Table II. The singlet parameters are the result of a $\chi^{2}$ search of $\beta$ and $\gamma$ over the exper-

TABLE I. Comparison of rank-2, UPA, and UPAA effective-range parameters.

\begin{tabular}{clcc}
\hline \hline Pot. & & $\begin{array}{c}-a_{t} \\
(\mathrm{~F})\end{array}$ & $\begin{array}{c}\boldsymbol{r}_{o t} \\
(\mathrm{~F})\end{array}$ \\
\hline \multirow{2}{*}{$X 1$} & Rank-2 & 1.8000 & 3.0710 \\
& UPA & 1.8031 & 3.0397 \\
& UPAA & 1.8025 & 3.0429 \\
$X 2$ & Rank-2 & 1.6000 & 3.1920 \\
& UPA & 1.6036 & 3.1524 \\
& UPAA & 1.6004 & 3.1797 \\
$X 3$ & Rank-2 & 1.4000 & 3.3476 \\
& UPA & 1.4024 & 3.2959 \\
& UPAA & 1.4000 & 3.3487 \\
\hline \hline
\end{tabular}


imental ${ }^{1} S_{0}$ phase shifts ${ }^{10}$ through a lab energy of $330 \mathrm{MeV}$, subject to the constraint that the experimental effective-range parameters, $a_{0 s}$ and $r_{0 s}$, are fitted exactly. The ${ }^{1} S_{0}$ phase shift for the potential shown in Table II changes sign at a lab energy of $278 \mathrm{MeV}$.

For the triplet $n-p$ parameters, we perform a least-squares scan of range parameters to fit the deuteron charge form factor, ${ }^{11}$ with the constraint that the triplet scattering length, $a_{t}$, and the deuteron binding energy ${ }^{12}$ are fitted exactly. The $n-p$ effective-range data employed, given by Houk and Wilson, ${ }^{13}$ are

$$
\begin{aligned}
& a_{s}=-23.714 \pm 0.013 \mathrm{~F}, \\
& a_{t}=5.425 \pm 0.005 \mathrm{~F}, \\
& r_{0 s}=2.704 \pm 0.075 \mathrm{~F} .
\end{aligned}
$$

The mixed triplet effective range ${ }^{13}$ quoted by Houk and Wilson is $\rho(0,-\epsilon)=1.763 \pm 0.005 \mathrm{~F}$, and is expressible in terms of the deuteron binding energy and $a_{t}$. Hence $\rho(0,-\epsilon)$ is fitted to high accuracy by virtue of the fit of $a_{t}$ and the deuteron binding energy. The resulting triplet effective range for the potential of Table II is found to be $r_{0 t}=1.787 \mathrm{~F}$. The $n-p$ parameters are chosen from a region of parameter space in which the repulsive singlet and triplet range parameters overlap, when a small latitude of $\chi^{2}$ to allow for experimental uncertainties is permitted.

An alternative shape of the repulsive form factor is the $\delta$ shell:

$$
f(k)=\sin (k \gamma) / k \gamma .
$$

The $\delta$-shell repulsion, considered previously by numerous workers, ${ }^{9,14}$ has the appealing feature of allowing a determination of its range in coordinate space. We find that although $\chi^{2}$ data fits are slightly improved upon adopting Eq. (20), the deuteron wave function is left with an unacceptable nodal structure. For this reason we do not employ the $\delta$ shell in this paper. Figure 1 shows a comparison between the deuteron wave functions using $f_{R}(k)$ given in Eqs. (18) and (20). Also shown is the rank-1 Yamaguchi deuteron wave function. The large loop in the $\delta$-shell deuteron wave function is

TABLE II. $n-p$ potential parameters. Parameters shown are for Yamaguchi attractive and repulsive form factors. The last two columns give the associated UPA parameters as defined in Sec. II.

\begin{tabular}{llrcccc}
\hline \hline & $\begin{array}{c}\lambda_{A} \\
\left(\mathrm{~F}^{-3}\right)\end{array}$ & $\begin{array}{c}\lambda_{R} \\
\left(\mathrm{~F}^{-3}\right)\end{array}$ & $\begin{array}{c}\beta \\
\left(\mathrm{F}^{-1}\right)\end{array}$ & $\begin{array}{c}\gamma \\
\left(\mathrm{F}^{-1}\right)\end{array}$ & $\begin{array}{c}\lambda_{u} \\
\left(\mathrm{~F}^{-3}\right)\end{array}$ & $X$ \\
\hline singlet & $\mathbf{0 . 3 6 0 7 9}$ & 3.2666 & 1.35 & 4.0 & 0.5057 & 2.086 \\
triplet & 2.3475 & 19.9793 & 1.88 & 4.0 & 4.4293 & 2.000 \\
\hline \hline
\end{tabular}

a direct consequence of the requirement that the deuteron charge form factor be fitted.

\section{THE SELF-CONSISTENT CALCULATION}

The $\Lambda$ binding in ${ }_{\Lambda} \mathrm{H}^{3}$ is more sensitive to the singlet than the triplet CS interaction. As shown in $I$, the particular interaction strength

$$
\lambda=\frac{3}{4} \lambda_{s}+\frac{1}{4} \lambda_{t},
$$

where $\lambda_{s}$ and $\lambda_{t}$ are the CS singlet and triplet $\Lambda-N$ strengths, is treated as an eigenvalue in the solution of the ${ }_{\Lambda} \mathrm{H}^{3}$ problem using separable (rank-1) potentials. In the same way, the rank- 2 potentials can be written as

$$
\left\langle\overrightarrow{\mathrm{k}}\left|V_{s}\right| \overrightarrow{\mathrm{k}}^{\prime}\right\rangle=-\left(\lambda_{s} / 2 \mu\right)\left[g_{A}(k) g_{A}\left(k^{\prime}\right)-R_{s} g_{R}(k) g_{R}\left(k^{\prime}\right)\right],
$$

where the subscript label $s$ denotes singlet. The triplet interaction is expressed in an identical fashion, with the use of the strengths $\lambda_{t}$ and $R_{t}$. $R_{s}$ and $R_{t}$ are given values prior to the self-consistent calculations, and the $\Lambda-N$ interaction strengths are determined by the requirements of self-consistency. For CSB potentials, $\epsilon_{s}$ and $\epsilon_{t}$ are used to denote the strengths corresponding to the CS strengths, $\lambda_{s}$ and $\lambda_{t}$.

As discussed in Sec. II, the attractive and repulsive strength parameters for the $N-N$ potentials (and thereby $R_{s}$ and $R_{t}$ ) are found by different criteria for the singlet than the triplet state. In the absence of form-factor and other high-energy $\Lambda-N$ data, we arbitrarily set $R_{s}=R_{t}=R$ in the $\Lambda-N$ interaction. A complete search in the full remain-

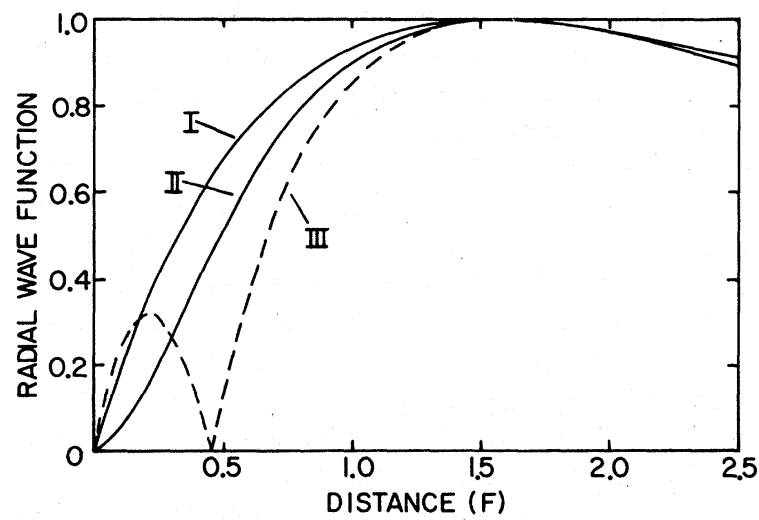

FIG. 1. Deuteron radial wave functions for different $n-p$ potentials. Curve $I$ is for the rank- 1 Yamaguchi triplet $n-p$ potential employed in earlier work (see paper I). Curve II is for the rank-2 potential employed in the present calculations. Curve III is for the $\delta$-shell repulsive term defined in Eq. (20). Potential I fits the scattering length and deuteron binding energy. Potentials II and III in addition are adjusted to fit the radial nucleon distribution obtained from Ref. 11. 
ing parameter space of the $\Lambda-N$ interactions for best data fits would seem supererogatory here. Our procedure, employing a more limited search, begins by setting the attractive singlet and triplet range parameter $\beta$ equal to $2.0 \mathrm{~F}^{-1}$. This corresponds to an intrinsic range of the attractive part of $3 / \beta$ or $1.5 \mathrm{~F}^{2} \mathrm{~A}$ search is then made for an optimum set of values for the common singlet and triplet range parameter, $\gamma$, and the over-all intrinsic range, $b$. Here, a lower bound to $\gamma$ of 3.7 $\mathrm{F}^{-1}$ is arbitrarily fixed. The associated intrinsic range is $0.81 \mathrm{~F}$. This analysis therefore assumes an upper limit on the repulsive range that would correspond approximately to the Yukawa intrinsic range for an exchanged $K$ meson. Larger values of $\gamma$ than $3.7 \mathrm{~F}^{-1}$ are found to give generally poorer $\chi^{2}$ fits to the $\sigma(\Lambda p)$ data. Consequently, $\gamma=3.7 \mathrm{~F}^{-1}$ is chosen for the entire analysis.

With $\beta$ and $\gamma$ specified, the ratio parameter $R$ is determined as a result of fixing the over-all intrinsic range, $b$. As in I, we find better fits to $\sigma(\Lambda p)$ and generally a better and more complete range of self-consistent solutions using $b=2.1 \mathrm{~F}$ than using $b=1.5 \mathrm{~F}$ or $1.8 \mathrm{~F}$. Consequently, in the remainder of the paper, only the case $b=2.1 \mathrm{~F}$ is discussed. The value $2.1 \mathrm{~F}$ is then used as a common intrinsic range for singlet and triplet, CS and CSB components in the $\Lambda-N$ interaction.

A scan of $\Lambda-p$ potentials is made over $0.1<-a_{s}$ $<6 \mathrm{~F}$ and $0.1<-a_{t}<3 \mathrm{~F}$ just as in $\mathrm{I}$, and the values of $\chi^{2}$ for the fit of the six $\sigma(\Lambda p)$ data points listed in Table I of I are determined. The $\Lambda-p$ over-all interaction strengths, as defined in Eq. (22), are the only remaining free parameters and are uniquely determined by $a_{s}$ and $a_{t}$. We label these $\Lambda-p$ strengths by $\lambda_{s x}$ and $\lambda_{t x}$. In order to be assured that the analytic behavior of our rank-2 potentials is actually producing a repulsion at sufficiently high energies, we calculate the phase shift of every acceptable $\Lambda-p$ potential obtained from fitting $\sigma(\Lambda p)$. In every case, the phase shift goes negative. For the values of $\beta, \gamma$, and $b$ employed, the $\Lambda-p$ phase shift changes sign near $E_{1 \mathrm{ab}}=120$ $\mathrm{MeV}$. The zero-crossing energy has only a slight dependence upon the values of the singlet and triplet scattering lengths which the $\Lambda-p$ potentials must fit in the data scan. The phase shift can be made to first become negative at higher energies by increasing the repulsive intrinsic range while keeping the over-all intrinsic range fixed, and then scanning over $a_{s}$ and $a_{t}$. However, there are not as yet any experimental data to allow this to be done in a meaningful manner.

Shown in Table III are a few of the $\Lambda-p$ potentials that satisfy the criterion $\chi^{2}-1.8 \leqslant 1.0$. As can be seen there, the best $\chi^{2}$ values are only slightly better than were obtained in I using rank-1 poten- tials, in spite of the additional two parameters ( $\gamma$ and $R$ ) employed in the fit. Table III illustrates the large spread in scattering-length ratios $a_{s} / a_{t}$ which is found among the excellent fits to $\sigma(\Lambda p)$. The best fit has the value of 0.95 for this ratio and $\chi^{2}=1.77$.

To best illustrate the nature of the self-consistent calculation, we use, as in I, the spin-dependence parameters $S, M, S_{x}$ defined by

$$
\begin{aligned}
& S=\frac{\sqrt{3}}{4} \frac{\lambda_{\sigma}}{\lambda}, M=\frac{\sqrt{3}}{4} \frac{\epsilon_{\sigma}}{\lambda}, \\
& S_{x}=\frac{\sqrt{3}}{4} \frac{\lambda_{s x}-\lambda_{t x}}{\lambda},
\end{aligned}
$$

where $\lambda_{\sigma}=\lambda_{s}-\lambda_{t}$ and $\epsilon_{\sigma}=\epsilon_{s}-\epsilon_{t}$. It follows that

$$
S_{x}=S+M \text {. }
$$

As discussed in I, self-consistency requires $S>0$ and either $S>M$ (for the case where the spin, $I$, of ${ }_{\Lambda} \mathrm{He}^{4}$ is assumed to equal 0 ) or $M>S$ (for $I=1$ ). Self-consistency does not require $S_{x}>0$ for the case $I=0$, since $M$ can be negative. It is therefore possible for the $\Lambda-p$ singlet interaction to be weaker than the $\Lambda-p$ triplet interaction and still satisfy self-consistency. Thus it is necessary to include in our scan scattering lengths for which $\left|a_{s} / a_{t}\right|<1$, and as shown in Table IV, self-consistency is sometimes attained when this inequality is met. This contrasts with the results of $I$, in which only positive values of $M$ were found and $\left|a_{s} / a_{t}\right|$ $>1$.

TABLE III. Selected $\Lambda-p$ potentials with intrinsic range of $2.1 \mathrm{~F}$.

\begin{tabular}{rcccccc}
\hline \hline & $b$ & & $-a_{s}$ & $-a_{t}$ & $r_{0 s}$ & $r_{0 t}$ \\
Pot. & $(\mathrm{F})$ & $\chi^{2}$ & $(\mathrm{~F})$ & $(\mathrm{F})$ & $(\mathrm{F})$ & $(\mathrm{F})$ \\
\hline 1 & 2.1 & 2.57 & 1.3 & 2.0 & 3.44 & 2.97 \\
2 & 2.1 & 2.04 & 1.4 & 2.1 & 3.35 & 2.93 \\
3 & 2.1 & 2.35 & 1.6 & 1.9 & 3.19 & 3.02 \\
4 & 2.1 & 1.77 & 1.8 & 1.9 & 3.07 & 3.02 \\
5 & 2.1 & 2.07 & 2.0 & 1.9 & 2.97 & 3.02 \\
6 & 2.1 & 2.45 & 2.2 & 1.7 & 2.89 & 3.13 \\
7 & 2.1 & 1.88 & 2.4 & 1.7 & 2.83 & 3.13 \\
8 & 2.1 & 2.42 & 2.4 & 1.8 & 2.83 & 3.07 \\
9 & 2.1 & 1.86 & 2.5 & 1.7 & 2.80 & 3.13 \\
10 & 2.1 & 2.39 & 2.6 & 1.6 & 2.77 & 3.19 \\
11 & 2.1 & 1.99 & 2.6 & 1.7 & 2.77 & 3.13 \\
12 & 2.1 & 2.26 & 2.7 & 1.7 & 2.75 & 3.13 \\
13 & 2.1 & 2.01 & 2.8 & 1.6 & 2.72 & 3.19 \\
14 & 2.1 & 2.66 & 2.8 & 1.7 & 2.72 & 3.13 \\
15 & 2.1 & 2.15 & 3.0 & 1.6 & 2.68 & 3.19 \\
16 & 2.1 & 2.26 & 3.2 & 1.5 & 2.65 & 3.26 \\
17 & 2.1 & 2.73 & 3.6 & 1.5 & 2.59 & 3.26 \\
18 & 2.1 & 2.80 & 4.0 & 1.4 & 2.54 & 3.35 \\
& & & & & & \\
\hline \hline
\end{tabular}


TABLE IV $\Lambda-N$ self-consistent potentials for $b=2.1 \mathrm{~F}$. Listed are all self-consistent solutions obtained using the $\Lambda-p$ potentials of Table III, while assuming spin 0 for ${ }_{\Lambda} \mathrm{He}^{4}$. The effects of the $T=1$ state in ${ }_{\Lambda} \mathrm{H}^{3}$ are included. Suffixes $A, B, C$ in column 1 denote $B_{\Lambda}=0.05$, 0.17 , and $0.25 \mathrm{MeV}$, respectively.

\begin{tabular}{|c|c|c|c|c|c|c|c|c|}
\hline Pot. & $M$ & $S$ & $\begin{array}{c}\lambda \\
\left(F^{-3}\right)\end{array}$ & $\begin{array}{c}\lambda_{B} \\
\left(F^{-3}\right)\end{array}$ & $\alpha$ & $r_{s x}$ & $r_{t x}$ & $r_{s t}$ \\
\hline $6 A$ & 0.024 & 0.028 & 1.32 & -0.019 & -5.1 & 0.10 & 0.057 & 2.0 \\
\hline $10 A$ & 0.043 & 0.056 & 1.32 & -0.032 & -4.2 & 0.15 & 0.080 & 2.3 \\
\hline $13 A$ & 0.051 & 0.062 & 1.32 & -0.039 & -4.0 & 0.17 & 0.090 & 2.3 \\
\hline $15 A$ & 0.060 & 0.066 & 1.32 & -0.045 & -3.8 & 0.19 & 0.100 & 2.4 \\
\hline $16 A$ & 0.065 & 0.087 & 1.32 & -0.049 & -3.8 & 0.19 & 0.11 & 2.5 \\
\hline $17 A$ & 0.078 & 0.095 & 1.31 & -0.059 & -3.6 & 0.22 & 0.12 & 2.5 \\
\hline $18 A$ & 0.087 & 0.12 & 1.31 & -0.066 & -3.6 & 0.24 & 0.14 & 2.6 \\
\hline $4 B$ & -0.018 & 0.008 & 1.40 & 0.015 & -0.14 & -0.034 & 0.009 & -3.7 \\
\hline $5 B$ & -0.006 & 0.016 & 1.41 & 0.005 & 5.6 & 0.009 & 0.030 & 3.9 \\
\hline $6 B$ & 0.001 & 0.049 & 1.41 & -0.001 & -72 & 0.030 & 0.032 & 1.1 \\
\hline $7 B$ & 0.011 & 0.055 & 1.41 & -0.009 & -7.8 & 0.060 & 0.044 & 1.6 \\
\hline $8 B$ & 0.013 & 0.042 & 1.41 & -0.010 & -7.0 & 0.067 & 0.045 & 1.7 \\
\hline $9 B$ & 0.015 & 0.058 & 1.41 & -0.012 & -6.3 & 0.073 & 0.049 & 1.8 \\
\hline $10 B$ & 0.017 & 0.075 & 1.41 & -0.014 & -5.9 & 0.078 & 0.053 & 1.8 \\
\hline $11 B$ & 0.020 & 0.061 & 1.41 & -0.016 & -5.6 & 0.086 & 0.055 & 1.9 \\
\hline $12 B$ & 0.024 & 0.064 & 1.41 & -0.019 & -5.1 & 0.097 & 0.060 & 2.0 \\
\hline $13 B$ & 0.026 & 0.080 & 1.41 & -0.021 & -4.9 & 0.10 & 0.063 & 2.0 \\
\hline $14 B$ & 0.028 & 0.066 & 1.41 & -0.023 & -4.8 & 0.11 & 0.064 & 2.1 \\
\hline $15 B$ & 0.033 & 0.085 & 1.41 & -0.027 & -4.5 & 0.12 & 0.073 & 2.1 \\
\hline $16 B$ & 0.037 & 0.10 & 1.41 & -0.030 & -4.3 & 0.13 & 0.080 & 2.2 \\
\hline $17 B$ & 0.050 & 0.11 & 1.40 & -0.040 & -4.0 & 0.16 & 0.096 & 2.3 \\
\hline $18 B$ & 0.058 & 0.13 & 1.40 & -0.047 & -3.8 & 0.17 & 0.11 & 2.4 \\
\hline $3 C$ & -0.040 & 0.008 & 1.44 & 0.034 & -1.7 & -0.12 & -0.017 & 6.6 \\
\hline $4 C$ & -0.027 & 0.017 & 1.45 & 0.023 & -1.1 & -0.068 & -0.002 & 39 \\
\hline $5 C$ & -0.016 & 0.025 & 1.45 & 0.013 & 0.30 & -0.025 & 0.012 & -2.1 \\
\hline $6 C$ & -0.009 & 0.057 & 1.45 & 0.008 & 2.6 & $-0.002^{-}$ & 0.020 & -0.11 \\
\hline $7 C$ & 0.001 & 0.064 & 1.45 & -0.0004 & -101 & 0.028 & 0.031 & 1.0 \\
\hline $8 C$ & 0.003 & 0.051 & 1.45 & -0.002 & -23 & 0.036 & 0.034 & 1.2 \\
\hline $9 C$ & 0.005 & 0.066 & 1.45 & -0.004 & -13 & 0.042 & 0.038 & 1.3 \\
\hline $10 C$ & 0.007 & 0.083 & 1.45 & -0.006 & -10 & 0.048 & 0.041 & 1.4 \\
\hline $11 C$ & 0.009 & 0.069 & 1.45 & -0.008 & -8.5 & 0.055 & 0.043 & 1.5 \\
\hline $12 C$ & 0.013 & 0.072 & 1.45 & -0.011 & -6.8 & 0.067 & 0.048 & 1.7 \\
\hline $13 C$ & 0.015 & 0.090 & 1.45 & -0.012 & -6.3 & 0.071 & 0.051 & 1.8 \\
\hline $14 C$ & 0.017 & 0.074 & 1.45 & -0.014 & -5.9 & 0.078 & 0.053 & 1.8 \\
\hline $15 C$ & 0.022 & 0.093 & 1.45 & -0.018 & -5.2 & 0.091 & 0.060 & 2.0 \\
\hline $16 C$ & 0.026 & 0.11 & 1.45 & -0.022 & -4.9 & 0.10 & 0.068 & 2.0 \\
\hline $17 C$ & 0.038 & 0.12 & 1.45 & -0.032 & -4.3 & 0.13 & 0.083 & 2.2 \\
\hline $18 C$ & 0.046 & 0.14 & 1.44 & -0.038 & -4.0 & 0.15 & 0.096 & 2.3 \\
\hline
\end{tabular}

As in I, self-consistency implies that the $\Lambda-N$ potential satisfies the inequalities just given, and also produces the same value of $\lambda$ and $M$ in the hypertriton calculation as in the fits to $\sigma(\Lambda p)$. These scattering-data fits are of course accompanied by the constraint upon the interaction parameters imposed by $\Delta=0.01$. The solutions of Table IV are classified according to which value for $B_{\Lambda}\left({ }_{\Lambda} \mathrm{H}^{3}\right)$ is assumed. The suffixes $A, B, C$ stand, respectively, for $B_{\Lambda}=0.05,0.17$, and $0.25 \mathrm{MeV}$. In the notation of $I$, the states $|+, 0\rangle$ and $|-, 1\rangle$ are included in the ${ }_{\Lambda} \mathrm{H}^{3}$ wave function, allowing in this manner for isospin mixing. Effects of isospin mixing for $I=0$ are shown in Table V. When a com- parison is made with Table VI of $\mathrm{I}$, the percentage effect of including the $T=1$ state $|-, 1\rangle$ in ${ }_{\Lambda} \mathrm{H}^{3}$ is seen to be quite similar. Isospin mixing has the effect in all cases of making $M$ more positive, or, in some cases in the rank-2 analysis, less negative, with a corresponding decrease of $S$ in all cases.

We define CSB fractions $r_{s x}$ and $r_{t x}$ by

$$
r_{s x}=\epsilon_{s} / \lambda_{s x}, \quad r_{t x}=\epsilon_{t} / \lambda_{t x} .
$$

We shall also employ the ratio $r_{s t} \doteq r_{s x} / r_{t x}$. The effects of isospin mixing tend to be more pronounced at $B_{\Lambda}=0.05 \mathrm{MeV}$, and at this energy serve to increase the CSB fractions. Figure 2 is a plot 
TABLE V. Effects of isospin mixing in ${ }_{\Lambda} \mathrm{H}^{3}$. Change of potential parameters upon inclusion of $T=1$ state, expressed in percent of values obtained when $T=1$ state is not included. Given to the nearest tenth of a percent.

\begin{tabular}{rcccccccc}
\hline \hline Pot. & $\begin{array}{c}M \\
(\%)\end{array}$ & $\begin{array}{c}S \\
(\%)\end{array}$ & $\begin{array}{c}\lambda \\
(\%)\end{array}$ & $\begin{array}{c}\lambda_{B} \\
(\%)\end{array}$ & $\begin{array}{c}\alpha \\
(\%)\end{array}$ & $\begin{array}{c}r_{s x} \\
(\%)\end{array}$ & $\begin{array}{c}r_{t x} \\
(\%)\end{array}$ & \multicolumn{1}{c}{$\begin{array}{r}r_{s t} \\
(\%)\end{array}$} \\
\hline $6 A$ & 0.8 & -0.4 & -0.1 & 0.8 & -0.4 & 0.6 & 0.4 & 0.2 \\
$10 A$ & 1.9 & -1.1 & -0.2 & 1.7 & -0.5 & 1.4 & 1.0 & 0.4 \\
$13 A$ & 2.4 & -1.3 & -0.3 & 2.1 & -0.6 & 1.8 & 1.3 & 0.4 \\
$15 A$ & 2.8 & -1.6 & -0.4 & 2.4 & -0.6 & 2.1 & 1.6 & 0.5 \\
$16 A$ & 3.0 & -1.4 & -0.4 & 2.5 & -0.6 & 2.2 & 1.7 & 0.5 \\
$17 A$ & 3.7 & -1.7 & -0.6 & 3.0 & -0.6 & 2.7 & 2.2 & 0.5 \\
$18 A$ & 4.1 & -1.6 & -0.8 & 3.3 & -0.6 & 3.0 & 2.4 & 0.5 \\
$3 C$ & -1.7 & -9.4 & -0.2 & 2.0 & -1.4 & -2.5 & -5.2 & 2.8 \\
$4 C$ & -1.5 & -2.3 & -0.1 & -1.6 & -2.4 & -2.2 & -21.9 & 25.3 \\
$5 C$ & -0.6 & -0.4 & 0 & -0.7 & 7.5 & -1.4 & 0.9 & -2.4 \\
$6 C$ & -1.1 & -0.2 & 0 & -1.1 & 2.4 & -13.9 & 0.6 & -14.4 \\
$7 C$ & 0 & 0 & 0 & 0 & -4.0 & -3.8 & -3.9 & 0.2 \\
$8 C$ & 0 & 0 & 0 & 0 & 0 & 0 & 0 & 0 \\
$9 C$ & 0 & 0 & 0 & 0 & 0 & 0 & 0 & 0 \\
$10 C$ & 0 & 0 & 0 & 0.7 & -0.5 & 0.3 & 0.1 & 0.2 \\
$11 C$ & 0 & -0.1 & 0 & 0.5 & -0.3 & 0.3 & 0.1 & 0.1 \\
$12 C$ & 0.7 & -0.1 & 0 & -0.7 & -0.4 & 0.4 & 0.2 & 0.2 \\
$13 C$ & 0.7 & -0.1 & 0 & 0.9 & -0.5 & 0.5 & 0.3 & 0.3 \\
$14 C$ & 1.1 & -0.1 & 0 & 1.1 & -0.6 & 0.7 & 0.4 & 0.3 \\
$15 C$ & 1.4 & -0.2 & -0.1 & 1.0 & -0.5 & 0.7 & 0.4 & 0.3 \\
$16 C$ & 1.2 & -0.2 & -0.1 & 1.2 & -0.5 & 0.9 & 0.6 & 0.3 \\
$17 C$ & 1.9 & -0.4 & -0.2 & 1.8 & -0.6 & 1.4 & 1.0 & 0.4 \\
$18 C$ & 2.2 & -0.4 & -0.2 & 1.9 & -0.5 & 1.6 & 1.2 & 0.4 \\
\hline \hline
\end{tabular}

of the CSB fractions as a function of the self-consistent value of the parameter $M$. Assumed here is that $I=0$. Figure 2 should be compared with Fig. 7 of I. The repulsions employed in the rank-2

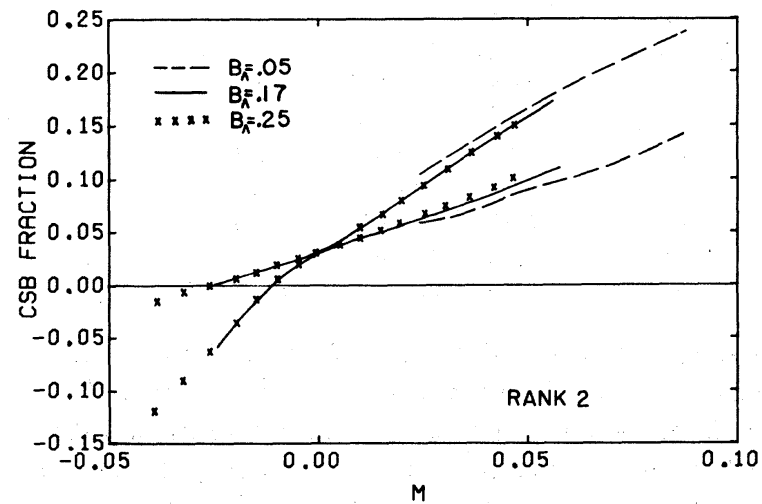

FIG. 2. CSB fractions for the rank-2 potentials as a function of the self-consistent value of the CSB spindifference parameter, $M$. Curves on upper right are singlet fractions $\left(r_{s x}\right)$ and curves on lower right are triplet fractions $\left(r_{t x}\right)$, as defined in Eq. (25). All curves cross at $M=0$. These are smooth curves drawn through a plot of all self-consistent CSB potentials in order to show the systematic variation with the assumed values of $B_{\Lambda}$. Comparison should be made with Fig. 7 of paper I. potential have the effect of permitting negative (repulsive) singlet and triplet CSB fractions, which is in closer qualitative agreement with Downs's $\mathrm{SU}_{3}$ theory of CSB.

The UPAA is employed in a parallel self-consistent calculation. Starting with the rank-2 $\Lambda-p$ po-

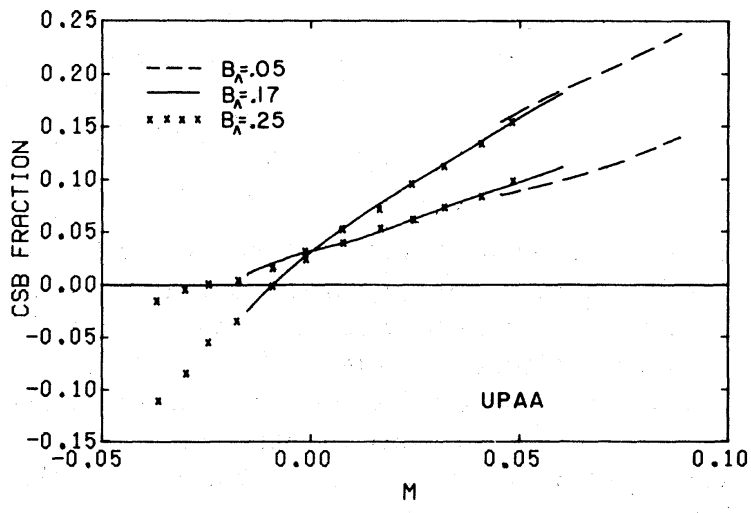

FIG. 3. CSB fractions for the UPAA approximation to the rank-2 potentials as a function of $\boldsymbol{M}$. Upper-right curves are singlet fractions $\left(\gamma_{s x}\right)$; lower-right curves are triplet fractions $\left(r_{t x}\right)$, as defined in Eq. (25). All curves cross at $M=0$. These curves again illustrate the variation of self-consistent CSB parameters with $B_{\Lambda}$, and the similarity to Fig. 2 should be noted. 
TABLE VI. $\Lambda-N$ self-consistent potentials for $I=1$. Listed are all self-consistent solutions obtained using the $\Lambda-p$ potentials of Table III, while assuming spin 1 for ${ }_{\Lambda} \mathrm{He}^{4}$. The effects of the $T=1$ state in $\mathrm{H}^{3}$ are included. The prefix $S$ is used in column 1 to identify potentials associated with $I=1$.

\begin{tabular}{rcccccccc}
\hline \hline Pot. & $M$ & $S$ & $\begin{array}{c}\lambda \\
\left(\mathrm{F}^{-3}\right)\end{array}$ & $\begin{array}{c}\lambda_{B} \\
\left(\mathrm{~F}^{-3}\right)\end{array}$ & $\alpha$ & $r_{s, x}$ & $r_{t x}$ & $r_{s t}$ \\
\hline$S 7 A$ & 0.036 & 0.035 & 1.32 & -0.027 & -4.4 & 0.13 & 0.069 & 2.2 \\
$S 9 A$ & 0.045 & 0.033 & 1.32 & -0.035 & -3.6 & 0.15 & 0.067 & 2.6 \\
$S 11 A$ & 0.055 & 0.031 & 1.32 & -0.042 & -3.1 & 0.16 & 0.065 & 2.9 \\
$S 12 A$ & 0.065 & 0.029 & 1.31 & -0.047 & -2.7 & 0.17 & 0.062 & 3.4 \\
$S 14 A$ & 0.074 & 0.026 & 1.31 & -0.056 & -2.4 & 0.19 & 0.059 & 3.9 \\
\hline \hline
\end{tabular}

tentials, we find the corresponding UPAA potentials. We take the shape of the CSB potential to be the same as the UPAA, and we find the function $\lambda(M)$ again for ${ }_{\Lambda} \mathrm{H}^{3}$. For $I=0$, the self-consistent results are shown in Fig. 3, which is similar to Fig. 2. The UPAA $\Lambda-N$ potentials do not possess strong repulsions to the extent of changing the sign of the phase shifts, as do the rank-2 potentials. However, they clearly manifest some of the characteristics of repulsions in that negative CSB fractions obtain at the largest $B_{\Lambda}$.

The sensitivity of the $\Lambda-N$ parameters to change of $\Delta$ is similar to what is shown in Table VII of I. This again indicates that our results are not significantly altered if $\Delta$ is allowed to vary by a few percent.

Finally, we list in Table VI the five $\Lambda-N$ potentials that survive the rigors of self-consistency when the spin of ${ }_{\Lambda} \mathrm{He}^{4}$ is assumed to be 1 . By the arguments presented in I, these potential parameters, with the larger values of $M$, are expected to be more sensitive to isospin mixing in ${ }_{\Lambda} \mathrm{H}^{3}$ than those for which $I=0$. Since the CSB fractions are all positive, we find a contradiction between Downs's $\mathrm{SU}_{3}$ prediction for the sign of the singlet CSB interaction and the assumption $I=1$, even when repulsions are included.

\section{DISCUSSION}

The repulsions used in this investigation are of primary importance in producing a number of low values of the CSB fractions. In the rank-1 calculations of I the CSB fractions were generally larger $(\sim 4 \%-25 \%)$ and all were positive. Although the present results for $B_{\Lambda}=0.05 \mathrm{MeV}$ are very similar in all respects to the results of $\mathrm{I}$, the higher values of $B_{\Lambda}, 0.17 \mathrm{MeV}$ and $0.25 \mathrm{MeV}$, produce radically different results. The combination of the repulsions and the higher binding energies yields solutions with CSB fractions that are quite small, and in some cases, negative. A negative CSB fraction corresponds to a CSB interaction which is repulsive in the $\Lambda-p$ state, and attractive in the $\Lambda-n$ state.
As already discussed in $\mathrm{I}$, the $\mathrm{SU}_{3}$ particle-mixing model of Downs predicts a repulsive singlet CSB interaction. Thus, we conclude that shortrange repulsions can be helpful in reconciling phenomenological requirements for CSB with meson theory.

Our best $\chi^{2}$ fits of $\sigma(\Lambda p)$ both in the rank-1 and rank-2 analyses occur for $\left|a_{s} / a_{t}\right| \leqslant 1$. As shown in I, many of these fits are rejected in the rank-1 case. In the present analysis, some of the best $\Lambda-p$ potentials for $\left|a_{s} / a_{t}\right| \leqslant 1$ give rise to self-consistent $\Lambda-N$ potentials with $M<0$ (consequently, as seen in Fig. 2, with $r_{s x}<0$ ). Hence repulsions play an important indirect role in allowing some of the best $\chi^{2}$ fits to $\sigma(\Lambda p)$ to be associated with selfconsistent potentials.

The results of the UPAA are of related importance. Here the effects of the repulsions in the rank-2 interactions are maintained. One observes, however, that the UPAA phase shifts are always positive. Thus, it is still not possible from these results to decide unequivocally between repulsions strong enough to cause the phase shifts to change sign, or simply a short-range weakening of the attraction.

A number of conclusions drawn in I still appear valid here. Isospin mixing in ${ }_{\Lambda} \mathrm{H}^{3}$ has the effect of causing, in some cases, a significant relative adjustment in the CSB parameters. As in I, the analysis illustrates the importance of retaining a spinindependent CSB term. In only a few isolated cases, for $B_{\Lambda}=0.17 \mathrm{MeV}$ and $0.25 \mathrm{MeV}$, are some values of $|\alpha|$ less than 1 encountered. For the range of values of $|\alpha|$ found, particularly for $B_{\Lambda}=0.05$ $\mathrm{MeV}$, there are considerable cancellations between the contributions of $\epsilon_{s}$ and $\epsilon_{t}$ to $\Delta$.

When the spin of ${ }_{\Lambda} \mathrm{He}^{4}$ is assumed to be 1 , only five self-consistent solutions are found. These solutions occur only for $b=2: 1 \mathrm{~F}$ and $B_{\Lambda}=0.05$ $\mathrm{MeV}$, with $a_{s} / a_{t}$ in the range 1.4-1.6. All five solutions have rather large CSB fractions: $v_{s x}$ $\sim 13 \%-19 \%, r_{t x} \sim 6 \%-7 \%$. Hence, if an experimental assignment of $I=1$ should be made, the present analysis would produce a highly constrained set of 
parameters for the $\Lambda-N$ CS and CSB potentials. However, only the value $I=0$ is consistent with the sign of the singlet CSB interaction predicted by the $\mathrm{SU}_{3}$ model of Downs.

No bound $\Lambda-N$ states occur with any of the potentials we obtain. In particular, for the $\Lambda-p$ potentials, the maximum well-depth parameter is 0.79 , while the best $\chi^{2}$ fit in our analysis, Pot. 4 , has singlet and triplet well-depth parameters of 0.54 and 0.55 , respectively.

Finally, there is a systematic variation of $\Lambda-N$ potential parameters with the spread of the available data. This is seen in Fig. 2. According to the present model, the $I=1$ state would be ruled out if $B_{\Lambda} \gg 0.05 \mathrm{MeV}$, while agreement with the sign of Downs's CSB singlet interaction would not occur if $B_{\Lambda} \ll<0.25 \mathrm{MeV}$. Improved low-energy $\Lambda-p$ cross sections should serve to decrease the number of acceptable $\Lambda-p$ potentials in a future analysis, while helping to specify the $\Lambda-p$ intrinsic range.

The introduction of the parameter $\Delta$ simplifies the analysis considerably. A future revision of the estimated CSB strength observed experimentally in ${ }_{\Lambda} \mathrm{H}^{4}$ and ${ }_{\Lambda} \mathrm{He}^{4}$ would simply affect the value of $\Delta$ employed in the self-consistent analysis of this paper. However, a more integrated approach to the problem of CSB is needed. The same potential model of baryon interactions should be used to extract $\Delta$ as to perform a self-consistent analysis using $\Lambda-p$ and ${ }_{\Lambda} \mathrm{H}^{3}$ data, and more complete fourbody data should be employed.
*Eppley Foundation for Research Scholar.

${ }^{1} \mathrm{~K}$. Hartt and E. Sullivan, preceding paper, Phys.

Rev. D 4 , 1353 (1971).

${ }^{2}$ Y. Yamaguchi, Phys. Rev. 95, 1628 (1954).

${ }^{3}$ B. W. Downs, Nuovo Cimento 43A, 454 (1966).

${ }^{4}$ C. Lovelace, Phys. Rev. 135, B1225 (1964).

${ }^{5}$ M. G. Fuda, Nucl. Phys. A116, 83 (1968); T. Brady, M. G. Fuda, E. Harms, J. S. Levinger, and R. Stagat, Phys. Rev. 186, 1069 (1969).

${ }^{6}$ G. Bohm et al., Nucl. Phys. B4, 511 (1968).

${ }^{7}$ R. C. Herndon and Y. C. Tang, Phys. Rev. 159, 853 (1967) (henceforth referred to as HT).
${ }^{8}$ G. Keys et al., Phys. Rev. D 1,67 (1970).

${ }^{9}$ F. Tabakin, Ann. Phys. (N.Y.) 30,51 (1964).

${ }^{10}$ M. MacGregor, Phys. Rev. 141,873 (1966).

${ }^{11}$ J. A. McIntyre and G. R. Burleson, Phys. Rev. 112, 2077 (1958)

${ }^{12}$ H. W. Taylor et al., Phys. Letters 24B, 659 (1967).

${ }^{13}$ T. L. Houk and R. Wilson, Rev. Mod. Phys. 39,546 (1967); 40 , 672(E) (1968).

${ }^{14}$ R. D. Puff, Ann. Phys. (N.Y.) 13, 317 (1961); F. Tabakin, Phys. Rev. 137, B75 (1963); J. Borysowicz and J. Dabrowski, Phys. Letters 24B, 125 (1967). 